\title{
The high energy emission line spectrum of NGC 1068
}

\author{
G. Matt ${ }^{1}$, S. Bianchi ${ }^{1}$, M. Guainazzi ${ }^{2}$, and S. Molendi ${ }^{3}$ \\ ${ }^{1}$ Dipartimento di Fisica, Università degli Studi Roma Tre, via della Vasca Navale 84, 00146 Roma, Italy \\ 2 XMM-Newton Science Operation Center/RSSD-ESA, Villafranca del Castillo, Spain \\ 3 IASF-CNR, Via Bassini 15, 20133, Milano, Italy
}

Received 21 August 2003 / Accepted 17 October 2003

\begin{abstract}
We present and discuss the high energy ( $E>4 \mathrm{keV})$ XMM-Newton spectrum of the Seyfert 2 galaxy, NGC 1068. Possible evidence for flux variability in both the neutral and ionized reflectors with respect to a BeppoSAX observation taken 3.5 years before is found. Several Fe and Ni emission lines, from both neutral and highly ionized material, are detected. The intensity of the iron $\mathrm{K} \alpha$ Compton shoulder implies that the neutral reflector is Compton-thick, likely the visible inner wall of the $N_{\mathrm{H}}>10^{25} \mathrm{~cm}^{-2}$ absorber. From the equivalent width of the ionized iron lines a column density of a few $\times 10^{21} \mathrm{~cm}^{-2}$ is deduced for the hot ionized reflector. Finally, an iron (nickel) overabundance, when compared to solar values, of about 2 (4) with respect to lower $Z$ elements, is found.
\end{abstract}

Key words. galaxies: individual: NGC 1068 - galaxies: Seyfert - X-rays: galaxies

\section{Introduction}

The archetypal Seyfert 2 galaxy, NGC 1068, has been extensively studied at all wavelengths. It was the discovery of broad lines in the polarized flux of this source that led Antonucci \& Miller (1985) to propose the Unification model for Seyfert galaxies, which is at present the most popular scenario for this class of sources. In X-rays, after the pioneering Einstein and EXOSAT observations (Monier \& Halpern 1987; Elvis \& Lawrence 1988), the GINGA discovery of a strong iron line (Koyama et al. 1989), being interpreted as due to the reprocessing by circumnuclear matter of the otherwise invisible nuclear radiation, brilliantly confirmed the Antonucci \& Miller model. ASCA (Ueno et al. 1994; Iwasawa et al. 1997; Bianchi et al. 2001) demonstrated that the line is complex, implying reflection from both neutral and ionized material. The two reflectors model was confirmed, observing the hard X-ray continuum, by BeppoSAX (Matt et al. 1997a), which was also able to put a lower limit of about $10^{25} \mathrm{~cm}^{-2}$ to the column density of the absorbing matter. Guainazzi et al. (1999) and Bianchi et al. (2001) demonstrated that the situation is even more complex, the line spectrum requiring at least three reflectors, one neutral (cold), one mildly ionized (warm) and one highly ionized (hot). Comparing the two BeppoSAX observations, taken about one year apart, Guainazzi et al. (2000) revealed a flux variability, which they interpreted as due to a variation of the intensity of the hot ionized reflector, so placing an upper limit to its size of the order of a parsec or so. Colbert et al. (2002), comparing ASCA, RXTE and BeppoSAX observations, found evidence

Send offprint requests to: $\mathrm{G}$. Matt, e-mail:matt@fis.uniroma3.it for an increase of the iron He-like line and a decrease of the continuum flux on time scale of months.

High spatial and spectral resolution Chandra and XMM-Newton observations have confirmed the complexity of the circumnuclear region. The Chandra image (Young et al. 2001) revealed, especially in soft $X$-rays, a very rich morphology, with the brightest spot, however, confined in a 1".5 (corresponding to $118 \mathrm{pc}$ assuming $H_{0}=70 \mathrm{~km} \mathrm{~s}^{-1} \mathrm{Mpc}^{-1}$, Young et al. 2001) region around the nucleus. Indeed, the iron emission as well as the neutral and ionized continuum reflection are mostly confined in the nuclear region (Ogle et al. 2003). Many off-center point-like sources are also detected (Smith \& Wilson 2003).

Gratings observations (Kinkhabwala et al. 2002; Ogle et al. 2003) have measured a line spectrum which is consistent with photoionized plasma with a wide range of ionization parameters. The line fluxes require significant contribution from resonant scattering (Kinkhabwala et al. 2002), as predicted by Band et al. (1990) and Matt et al. (1996). Given the limited energy range of the XMM-Newton/RGS and the modest effective area of the Chandra/HETG at high energies, these results mainly concern the soft X-ray line spectrum.

In this paper we analyse and discuss the high energy ( $>4 \mathrm{keV}$ ) XMM-Newton spectrum of NGC 1068. While the CCDs of the EPIC cameras have a significantly poorer energy resolution than the Chandra HETG (the only grating instrument currently working at the iron $\mathrm{K} \alpha$ energy), for many purposes the much larger effective area above $4 \mathrm{keV}$ overcompensate for the lower resolution. As an example, one can compare the wealth of informations on $\mathrm{Fe}$ and $\mathrm{Ni} \mathrm{K}$ lines derived from the XMM-Newton observation of the Circinus 

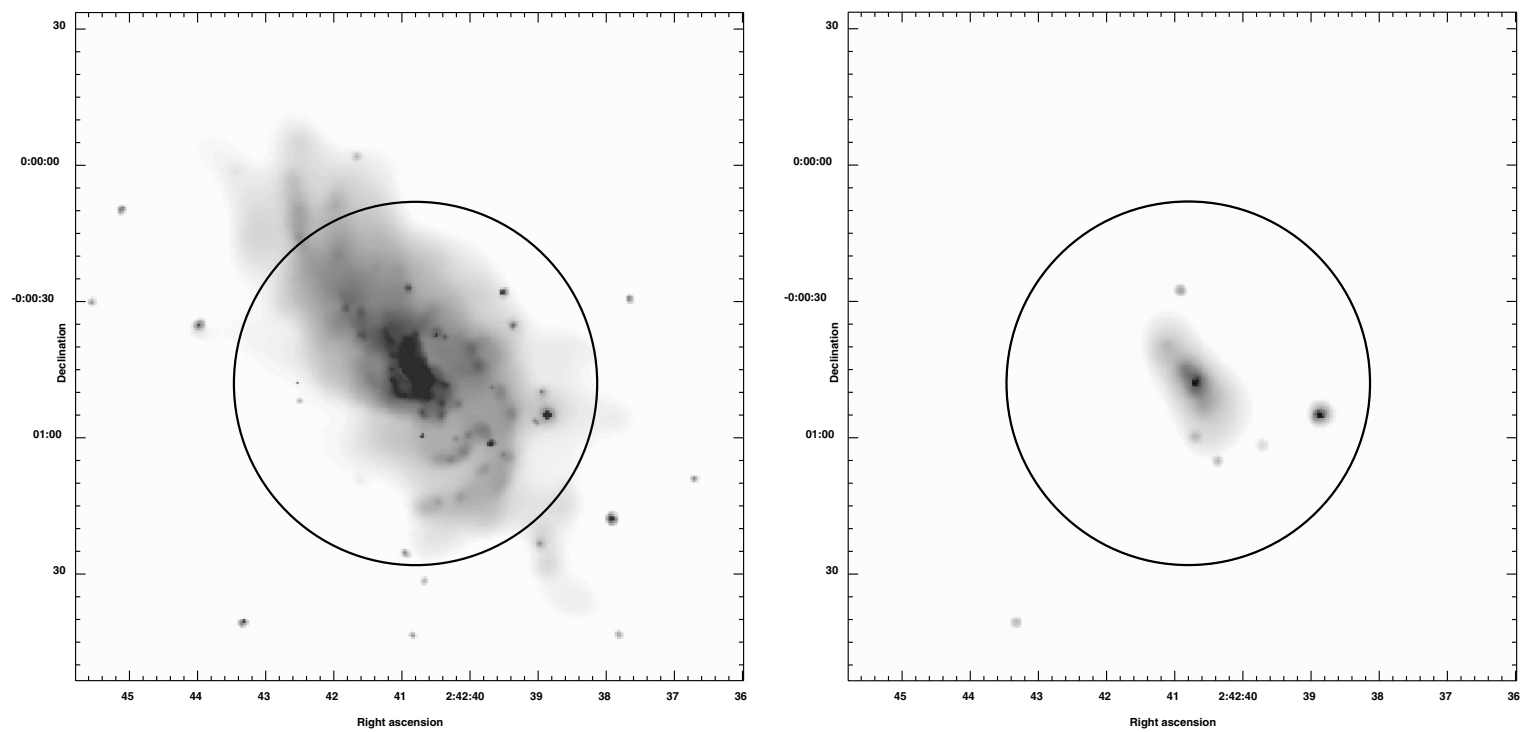

Fig. 1. Left: the whole band Chandra ACIS image of the nuclear region of NGC 1068. Right: the same, but only above 4 keV. Note that the extended emission is less prominent, and that one off-center source, CXJ024238.9-000055, becomes by far the most prominent one.

Galaxy (Molendi et al. 2003) with the much poorer informations on the same lines (indeed many of them not even detected) derived from the Chandra/HETG spectrum (Sambruna et al. 2001).

\section{Data reduction}

\section{1. $X M M-N e w t o n$}

NGC 1068 was observed twice by XMM-Newton on July 29, 2000 and July 30, 2000 with the imaging CCD cameras, the EPIC-pn and MOS, adopting the Medium filter and operating in Large Window (pn), Full Window (MOS1) and Prime Partial W2 (MOS2). We retrieved the ODF files from the public archive, and reduced them with the latest available SAS version (5.4.1), therefore using the most updated calibrations. The observed count rate is lower than the maximum defined for a $1 \%$ pileup for the selected pn subarray (see Table 3 of XMM-Newton Users' Handbook), but it is close to that value, so we conservatively decided to exclude patterns higher than 0 in data reduction. An analysis with the EPATPLOT tool indicates that same residual pile-up effect may still be present, so we extracted a spectrum removing the inner 6 " circle region. No significant differences are found, however, in the continuum and in the lines alike, if the comparison is limited to the energy range of interest here, i.e. above $4 \mathrm{keV}$. We therefore concluded that, for the pn, pile-up is not relevant for our purposes. On the other hand, we shall not use MOS data because MOS1 has been used in Full Window mode and therefore strongly suffers from pile-up problems, while MOS2 does not add much information, expecially because we will limit our analysis to $E>4 \mathrm{keV}$. Moreover, the pn appears to be the best calibrated detector at the energies under consideration, as deduced from the analysis of the iron line complex in the spectrum of the Circinus Galaxy (Molendi et al. 2003).
Since the two observations are nearly contiguous and no significant flux variations are found in their lightcurve, we verified that the two spectra were consistent with each other and decided to combine them. We used the SAS tool MERGE to obtain a single event file and then extracted a spectrum from a region of $40^{\prime \prime}$ radii. The total exposure time, after rejecting time intervals of flaring particle background, is $61 \mathrm{ks}$. Spectra were analysed with XSPEC 11.2.0.

\subsection{Chandra}

Given the complexity of the morphology of the circumnuclear region of NGC 1068, we also analysed the Chandra observation of NGC 1068 performed on February 21, 2000 with the Advanced CCD Imaging Spectrometer (ACIS-S), for a total exposure time of $48 \mathrm{ks}$. The default $3.2 \mathrm{~s}$ frame time, though resulting in the nuclear spectrum spoiled by pileup, does not affect our qualitative imaging analysis and the spectrum of the much fainter source CXJ024238.9-000055 (see below). Data were reduced with CIAO 2.3, using CALDB 2.21. Spectra were analysed with XSPEC 11.2.0.

\section{Spatial analysis}

In Fig. 1 the Chandra image over the whole working band (left panel) and above $4 \mathrm{keV}$ only (right panel) is shown. The detailed spatial analysis is discussed by Young et al. (2001), while the X-ray source population by Smith \& Wilson (2003). The XMM-Newton image (whole band, left panel, and above $4 \mathrm{keV}$, right panel) is shown in Fig. 2. In all images, the $r=40^{\prime \prime}$ circle over wich the XMM-Newton spectrum has been extracted is shown.

For our own purposes, it is important to note that: a) the nucleus is partly extended over several arcseconds even above $4 \mathrm{keV}$, the extended emission accounting for about a 

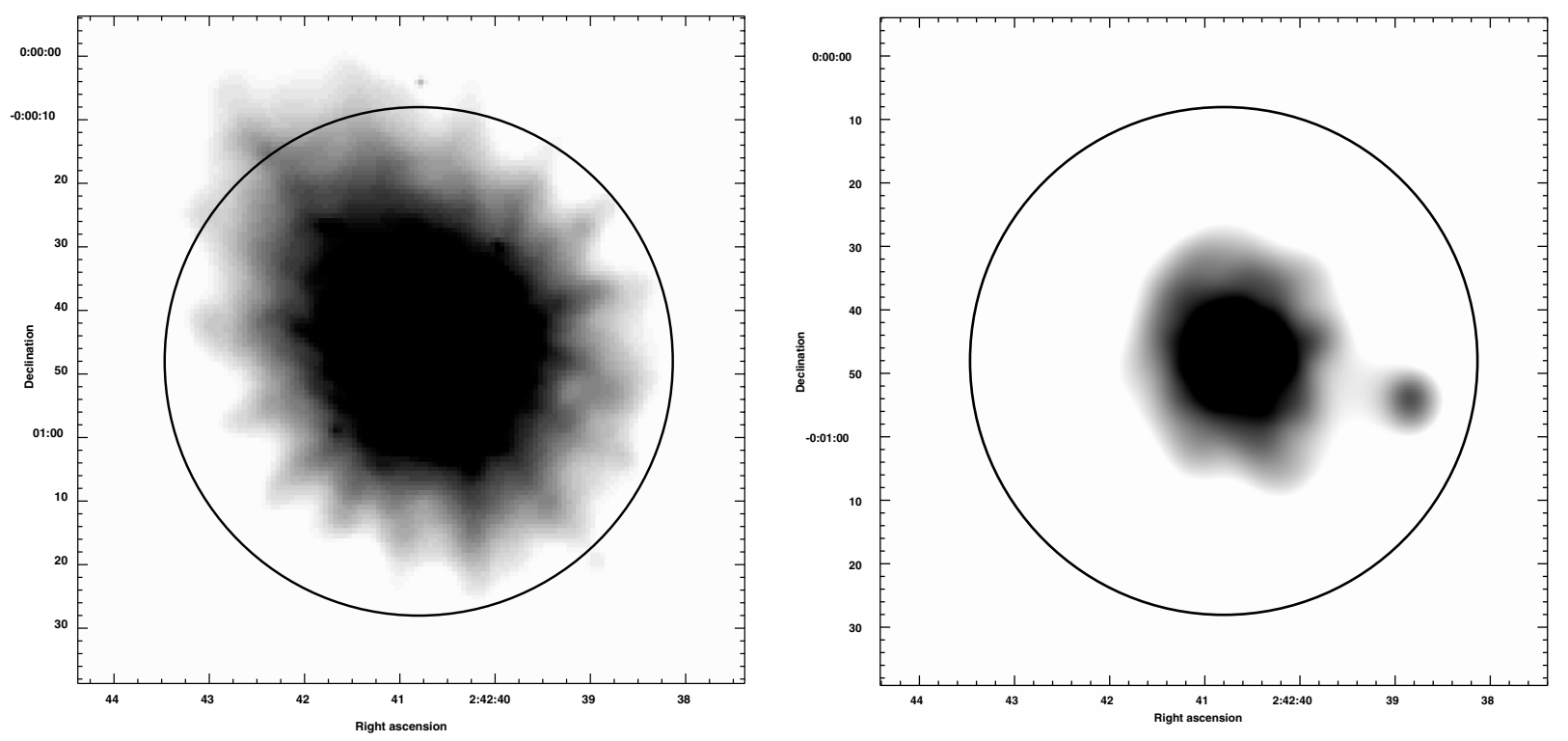

Fig. 2. Left: the whole band XMM-Newton EPIC-pn image of the nuclear region of NGC 1068. Right: the same, but only above 4 keV.

quarter of counts in this band. Our 40" XMM-Newton extraction regions encompasses this extended emission. b) At a distance of about 28", a bright point-like source (CXJ024238.9000055) is clearly apparent in the Chandra image (hereinafter we refer to it for simplicity as $\mathrm{X}-1$ ); in the hard band, it is by far the brightest off-nuclear point-like source. Smith \& Wilson (2003) derived for this source a $0.4-8 \mathrm{keV}$ flux of about $5 \times 10^{-13} \mathrm{erg} \mathrm{cm}^{-2} \mathrm{~s}^{-1}$, corresponding to a luminosity, at the distance of NGC 1068, of $1.4 \times 10^{40} \mathrm{erg} \mathrm{s}^{-1}$. The spectrum (which we reanalysed) is quite hard, being consistent with either a very flat $(\Gamma=0.85)$ power law or with multicolor disc blackbody with an inner temperature of about $4 \mathrm{keV}$. While we defer the reader to Smith \& Wilson (2003) for a discussion of its possible nature, we note here that this source, being so hard, may significantly contaminate the spectrum of the NGC 1068 nucleus in the energy range under consideration in this paper. Indeed, while the source is not readily visible in the whole band XMM-Newton image, it is clearly present in the $E>4 \mathrm{keV}$ image. To evaluate its contribution to the overall spectrum, we extracted a $20^{\prime \prime}$ spectrum centered on NGC 1068 and a $10^{\prime \prime}$ spectrum centered on $\mathrm{X}-1$. The flux we derived for NGC 1068 is 14 times larger than that of $\mathrm{X}-1$ (which in turn is about half that measured by Chandra, Smith \& Wilson 2003), and this is certainly a lower limit as the spectrum of $X-1$ is heavily contaminated by that of NGC 1068. In fact, in the $\mathrm{X}-1$ spectrum a broad iron line is clearly present, well fitted by two narrow components, one at $6.4 \mathrm{keV}$ (neutral iron) and one at $6.7 \mathrm{keV}$ (He-like iron). If we assume that they are the result of the contamination by NGC 1068 (no iron lines are apparent in the, admittedly poor, Chandra spectrum), this can provide a method to estimate the level of contamination of $\mathrm{X}-1$ by the nucleus. The EWs for these two lines are about 2.5 smaller than in the NGC 1068 spectrum (when calculated with respect to the whole continuum; see next section). Therefore, about $40 \%$ of the spectrum extracted around $X-1$ is actually due to NGC 1068, which implies that $\mathrm{X}-1$ does not contaminate the $40^{\prime \prime}$ spectrum of NGC 1068 by more than about $5 \%$. Considering also that we are mostly interested in the emission lines, which are unlikely to be produced by $\mathrm{X}-1$, we decided that this contamination is not large enough to justify the $20 \%$ loss in photons when using the $r=20^{\prime \prime}$ region. In the following we will then use the $40^{\prime \prime}$ radius spectrum, unless explicitely stated.

\section{The $E>4 \mathrm{keV} X M M-$ Newton spectrum}

The soft X-ray high energy resolution line spectrum of NGC 1068 is discussed in detail by Kinkhabwala et al. (2002) and Ogle et al. (2003). We limit ourselves to analyse and discuss the high energy $(E>4 \mathrm{keV})$, moderate resolution EPIC-pn spectrum of the nuclear region of NGC 1068, with particular emphasis on the iron and nickel line properties. Let us, however, first briefly discuss the continuum properties.

\subsection{The continuum}

Following Iwasawa et al. (1997) and Matt et al. (1997a), we fitted the continuum with two components, describing reflection from both neutral and ionized matter (the latter component including both the "warm" and "hot" regions, Bianchi et al. 2001). For the neutral reflection component we adopted, as customary, the model PEXRAV, with $R$ fixed to -1 (i.e. with the primary emission shut off). The ionized reflector component is instead modeled by a simple power law, as the ionized reflector should act as a "mirror" for the primary emission. The power law index has been assumed to be the same for the two models. To fit the spectrum, we of course added as many lines as required, as discussed in details in the next paragraphs. The fit is fully acceptable $\left(\chi^{2} /\right.$ d.o.f. $\left.=143.7 / 149\right)$. The best fit parameters for the continua, for the neutral and 
Table 1. Best fit results. Continua.

\begin{tabular}{lc}
\hline \hline$\Gamma$ & $2.04_{-0.03}^{+0.04}$ \\
$N_{\text {cold }}(1 \mathrm{keV})\left[\mathrm{ph} \mathrm{keV}^{-1} \mathrm{~cm}^{-2} \mathrm{~s}^{-1}\right]$ & 0.019 \\
$N_{\text {ion }}(1 \mathrm{keV})\left[\mathrm{ph} \mathrm{keV}^{-1} \mathrm{~cm}^{-2} \mathrm{~s}^{-1}\right]$ & $7.37 \times 10^{-4}$ \\
$F_{\text {cold }}(4-10 \mathrm{keV})\left[\mathrm{erg} \mathrm{cm}^{-2} \mathrm{~s}^{-1}\right]$ & $1.52 \times 10^{-12}$ \\
$F_{\text {ion }}(4-10 \mathrm{keV})\left[\mathrm{erg} \mathrm{cm}^{-2} \mathrm{~s}^{-1}\right]$ & $1.01 \times 10^{-12}$ \\
$\mathrm{~A}_{\mathrm{Fe}}^{a}$ & $2.39_{-0.36}^{+0.43}$ \\
\hline
\end{tabular}

${ }^{a}$ In solar units (Anders \& Grevesse 1989) by number.

the ionized lines are reported in Tables 1,2 and 3, respectively (all errors refer to $90 \%$ confidence level for one interesting parameter, unless explicitely stated). The folded spectrum and best fit model, along with the model/data ratio, are shown in Fig. 3, while the unfolded spectrum is shown in Fig. 4. The power law index is $2.04_{-0.03}^{+0.04}$, and the ratio between the 4-10 keV cold and ionized reflectors fluxes is about 1.5. (Fitting the spectrum extracted from a $20^{\prime \prime}$ radius, a slightly steeper index, $2.10_{-0.04}^{+0.04}$, is found; the ratio between the two components does not change significantly. All other parameters are also consistent with those obtained with the $r=40^{\prime \prime}$ extraction radius, but with of course somewhat larger error bars.) This result could be compared with that derived by Matt et al. (1997a) from the December 1996 BeppoSAX observation, where the flux ratio was instead 0.5 , suggesting significant variations in likely both components. Indeed, variability on a time scale of 1 year, probably in the ionized reflector component, has been reported by Guainazzi et al. (2000). However, the BeppoSAX result must be taken with caution: the $F_{\text {cold }} / F_{\text {ion }}$ ratio is very sensitive to the power law index, which in BeppoSAX was only loosely determined $\left(1.74_{-0.56}^{+0.25}\right)$. We re-fitted the BeppoSAX spectrum imposing the power law index found from XMM-Newton, i.e. 2.04. The result is only slightly worse statistically $\Delta \chi^{2}=2.7$ with one less free parameter, corresponding to a $90 \%$ confidence level according to the F-test), and the ratio now becomes $\sim 1.3$. On the contrary, fitting the XMM-Newton spectrum imposing the BeppoSAX best fit power law index gives a significantly worst fit $\left(\chi^{2} /\right.$ d.o.f. $\left.=174.4 / 150\right)$; the ratio is now about 1.15 . Indeed, the XMM-Newton $90 \%$ confidence level lower limit on $\Gamma$ (i.e. 2.01) corresponds to a flux ratio of 1.49. At this confidence level, therefore, the BeppoSAX and XMM-Newton estimates are inconsistent with each other. There is therefore some, even if still not conclusive given also the uncertainties when comparing different instruments, evidence for variability between the BeppoSAX and the XMM-Newton observations, taken 3.5 years apart, which would give an upper limit to the distance of the spatially unresolved reflecting regions of the order of a few parsecs. Clearly, a second XMM-Newton observation is necessary to confirm this finding.

Another interesting result is the large iron abundance, $A_{\mathrm{Fe}}=$ 2.4 (with respect to the solar value of Anders \& Grevesse 1989). In the fit, $A_{\mathrm{Fe}}$ is basically derived from the depth of the
Table 2. Best fit results. Lines from cold matter. Equivalent widths are calculated against the cold reflector only.

\begin{tabular}{lc}
\hline \hline$E(\mathrm{Fe} \mathrm{K} \alpha$ core $)[\mathrm{keV}]$ & $6.424_{-0.001}^{+0.001}$ \\
$F(\mathrm{Fe} \mathrm{K} \alpha$ core $)\left[10^{-6} \mathrm{ph} \mathrm{cm}^{-2} \mathrm{~s}^{-1}\right]$ & $44.3_{-3.0}^{+2.3}$ \\
$E W(\mathrm{Fe} \mathrm{K} \alpha$ core $)[\mathrm{eV}]$ & 1200 \\
$F(\mathrm{Fe} \mathrm{K} \alpha \mathrm{CS})\left[10^{-5} \mathrm{ph} \mathrm{cm}^{-2} \mathrm{~s}^{-1}\right]$ & $8.7_{-2.7}^{+1.3}$ \\
$E(\mathrm{Fe} \mathrm{K} \beta)[\mathrm{keV}]$ & $7.077_{-0.045}^{+0.003}$ \\
$F(\mathrm{Fe} \mathrm{K} \beta)\left[10^{-6} \mathrm{ph} \mathrm{cm}^{-2} \mathrm{~s}^{-1}\right]$ & $9.1_{-1.1}^{+2.1}$ \\
$E W(\mathrm{Fe} \mathrm{K} \beta)[\mathrm{eV}]$ & 346 \\
$E(\mathrm{Ni} \mathrm{K} \alpha)[\mathrm{keV}]$ & $7.48_{-0.05}^{+0.01}$ \\
$F(\mathrm{Ni} \mathrm{K} \alpha)\left[10^{-5} \mathrm{ph} \mathrm{cm}^{-2} \mathrm{~s}^{-1}\right]$ & $5.6_{-1.0}^{+1.8}$ \\
$E W(\mathrm{Ni} \mathrm{K} \alpha)[\mathrm{eV}]$ & 410 \\
$E(\mathrm{Ni} \mathrm{K} \beta)[\mathrm{keV}]$ & $8.25_{-0.03}^{+0.10}$ \\
$F(\mathrm{Ni} \mathrm{K} \beta)\left[10^{-5} \mathrm{ph} \mathrm{cm}{ }^{-2} \mathrm{~s}^{-1}\right]$ & $3.2_{-1.5}^{+1.1}$ \\
$E W(\mathrm{Ni} \mathrm{K} \beta)[\mathrm{eV}]$ & 230 \\
\hline
\end{tabular}

Table 3. Best fit results. Lines from ionized matter. Equivalent widths are calculated against the ionized reflector only.

\begin{tabular}{|c|c|}
\hline$E(\mathrm{Fe} \mathrm{Be}-\mathrm{like} \mathrm{K} \alpha)[\mathrm{keV}]$ & $6.61_{-0.04}^{+0.01}$ \\
\hline$F($ Fe Be-like K $\alpha)\left[10^{-5} \mathrm{ph} \mathrm{cm}^{-2} \mathrm{~s}^{-1}\right]$ & $7.6_{-1.1}^{+2.4}$ \\
\hline$E W(\mathrm{Fe} \mathrm{Be}-$ like $\mathrm{K} \alpha)[\mathrm{eV}]$ & 485 \\
\hline$E(\mathrm{Fe} \mathrm{He}-\mathrm{like} \mathrm{K} \alpha)[\mathrm{keV}]$ & $6.725_{-0.001}^{+0.001}$ \\
\hline$F(\mathrm{Fe} \mathrm{He}-$ like $\mathrm{K} \alpha)\left[10^{-5} \mathrm{ph} \mathrm{cm}^{-2} \mathrm{~s}^{-1}\right]$ & $21.8_{-1.4}^{+3.2}$ \\
\hline$E W(\mathrm{Fe} \mathrm{He}-\mathrm{like} \mathrm{K} \alpha)[\mathrm{eV}]$ & 1430 \\
\hline$E(\mathrm{Fe} \mathrm{H}-$ like $\mathrm{K} \alpha)[\mathrm{keV}]$ & $6.92_{-0.04}^{+0.01}$ \\
\hline$F(\mathrm{Fe} \mathrm{H}$-like $\mathrm{K} \alpha)\left[10^{-5} \mathrm{ph} \mathrm{cm}^{-2} \mathrm{~s}^{-1}\right]$ & $7.1_{-0.9}^{+2.8}$ \\
\hline$E W(\mathrm{Fe} \mathrm{H}-$ like $\mathrm{K} \alpha)[\mathrm{eV}]$ & 494 \\
\hline$E(\mathrm{Ni} \mathrm{He}-\mathrm{like} \mathrm{K} \alpha)[\mathrm{keV}]$ & $7.83_{-0.05}^{+0.04}$ \\
\hline$F(\mathrm{Ni} \mathrm{He}-$ like $\mathrm{K} \alpha)\left[10^{-5} \mathrm{ph} \mathrm{cm}^{-2} \mathrm{~s}^{-1}\right]$ & $2.7_{-1.3}^{+1.2}$ \\
\hline$E W(\mathrm{Ni} \mathrm{He}-\mathrm{like} \mathrm{K} \alpha)[\mathrm{eV}]$ & 246 \\
\hline
\end{tabular}

iron edge in the Compton reflection component, which depends on the spectral index and, for a plane-parallel geometry, also on the inclination angle. Solar abundances for the other elements (in particular for CNO elements, which contribute most to the photoelectric cross section at $6.4 \mathrm{keV}$ ) have been assumed. The fit has been performed keeping the inclination angle $i$ fixed to $63^{\circ}$, i.e. the XSPEC default value. For lower values of the inclination angle a less deep edge is expected, and therefore an even larger value of the iron abundance is found (e.g. 3.3 for an inclination of about $20^{\circ}$ ). In Fig. 5 the $A_{\mathrm{Fe}}-\Gamma$ (with $i$ free to vary) is shown; it is important to note that the iron abundance is larger than 1.5 at the $99 \%$ confidence level (two interesting parameters). 


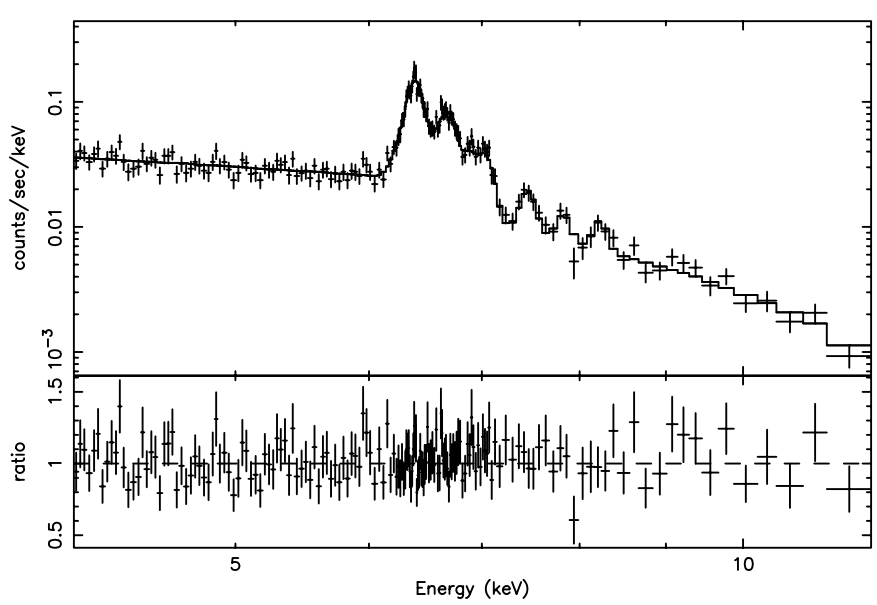

Fig. 3. Spectrum and best fit model, and model/data ratio. See text for details.

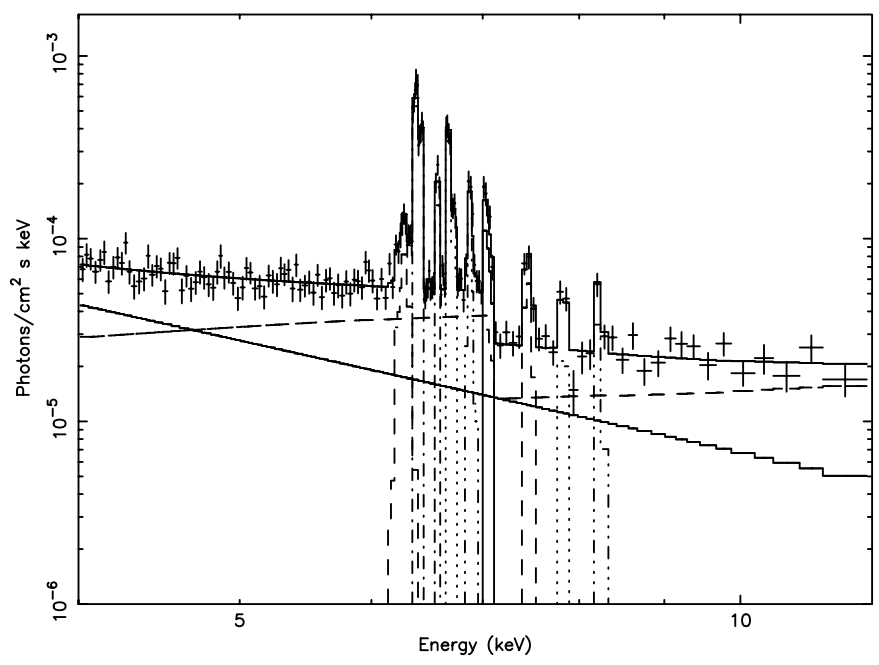

Fig. 4. Unfolded spectrum and best fit model. See text for details.

\subsection{Emission lines from the cold reflector}

Of the several lines observed in the high energy spectrum of NGC 1068 (see Fig. 3), four can be attributed to neutral Fe and $\mathrm{Ni}$ atoms, i.e. the $\mathrm{K} \alpha$ and $\mathrm{K} \beta$ lines from both elements (see Table 2). All lines, both neutral and ionized, have been fitted with a Gaussian profile with $\sigma$ fixed to $1 \mathrm{eV}$ (i.e. much less than the energy resolution; no improvement is found leaving $\sigma$ free to vary for the brigthest lines). All lines we attribute to the neutral reflector are significant at more than $99.99 \%$ confidence level, according to the $F$-test, apart from the $\mathrm{Ni} \mathrm{K} \beta$ at $8.25 \mathrm{keV}$ which is significant at the $99.90 \%$ confidence level.

The iron $\mathrm{K} \alpha$ line energy $(6.424 \pm 0.001 \mathrm{keV}$; note that the statistical error is much lower than the calibration uncertainty, which is of the order of $10 \mathrm{eV}^{1}$ ) is larger than the value for neutral iron (actually a doublet with energies of 6.391 and $6.405 \mathrm{keV}$, House 1969, with a 1:2 branching ratio), and would instead suggest Fe XVI; however, the measured $\mathrm{K} \beta / \mathrm{K} \alpha$ flux ratio for iron (including only the line core for the $\mathrm{K} \alpha$ line, see below) is $0.20_{-0.03}^{+0.07}$, slightly larger than

\footnotetext{
1 See http://xmm.vilspa.esa.es/docs/documents/ CAL-TN-0018-2-1.pdf
}

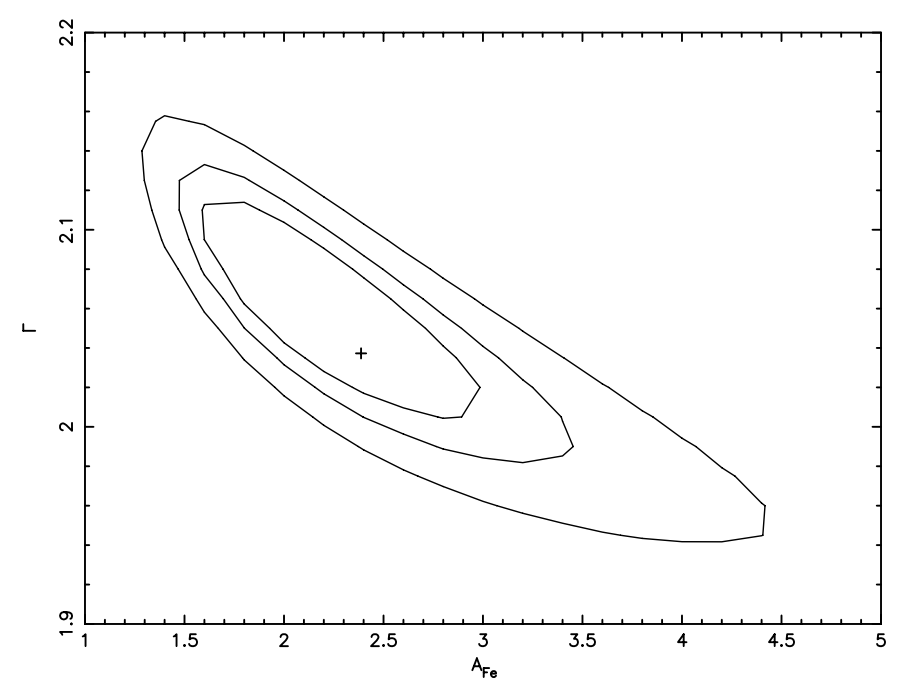

Fig. 5. $A_{\mathrm{Fe}}-\Gamma$ contour plot.

the expected value even for neutral value, which is about 0.16 (see Molendi et al. 2003). This value stays almost constant up to Fe IX, then starts decreasing (Kaastra \& Mewe 1993). It is therefore unlikely that iron is much more ionized than that (the line energy is also basically constant over this ionization range, House 1969). The discrepancy with the observed line energy may result from a less than perfect energy calibration of the pn, or a real blueshift of the line, which is however hard to explain in the classical torus model for the neutral reflection. It is worth noting that the MOS2 gives exactly the same best fit line energy, and that Chandra/HETG also measured a blueshift, albeit smaller $(6.411 \pm 0.007 \mathrm{keV}$, Ogle et al. 2003). The $\mathrm{K} \beta / \mathrm{K} \alpha$ ratio for nickel is $0.57 \pm 0.35$, definitely larger than the expected value, which is similar to that for iron (Kaastra \& Mewe 1993). While the measured energy of the line, $8.25_{-0.03}^{+0.10} \mathrm{keV}$, is perfectly consistent with the expected value of $8.265 \mathrm{keV}$ for the neutral Ni K $\beta$ line (Bearden 1967), the Fe XXVI K $\beta$ lines at energies clustering around $8.25^{2}$ are also possibly contributing.

The Ni-to-Fe $\mathrm{K} \alpha$ flux ratio is $0.13 \pm 0.03$, much larger than the expected value of $0.04-0.06$ (calculated as described in Molendi et al. 2003 assuming a factor 2 overabundance for both iron and nickel). This implies a nickel-to-iron overabundance of a factor $\sim 2$, or more

Clear evidence for an iron $\mathrm{K} \alpha$ Compton shoulder (hereinafter CS) is also found, confirming and refining the ASCA (Iwasawa et al. 1997) and Chandra/HETG (Ogle et al. 2003) results. The CS is fitted with a Gaussian profile with the centroid energy and $\sigma$ fixed to $6.3 \mathrm{keV}$ and $40 \mathrm{eV}$, respectively (Matt 2002). If the CS is not included a significantly worse fit $\left(\chi^{2} /\right.$ d.o.f. $\left.=181.2 / 149\right)$ is found, even allowing for the width of the main line to vary. The ratio between the CS and the line core fluxes is about 0.2 , in agreement with the theoretical expectation for Compton-thick reflecting material (Matt 2002), so suggesting an origin in the visible part of the $N_{\mathrm{H}}>10^{25} \mathrm{~cm}^{-2}$ absorbing material (Matt et al. 1997a).

\footnotetext{
${ }^{2}$ Unless explicitely stated, line energies are taken from the NIST Atomic Spectra Database,

http://physics.nist.gov/cgi-bin/AtData/main_asd
} 
The iron $\mathrm{K} \alpha E W$ (with respect to the cold reflection continuum) is $1.2 \mathrm{keV}$ ( $1.45 \mathrm{keV}$ including the CS), reasonably in agreement with theoretical expectations (e.g. Ghisellini et al. 1994; Matt et al. 2003) even with the observed iron overabundance, given the less than linear increase of the line $E W$ with the iron abundance (Matt et al. 1997b).

\subsection{Emission lines from the ionized reflector}

Four emission lines from highly ionized matter (the "hot" reflector cited above) are also detected in the pn spectrum (Table 3). All of them are significant at more than $99.99 \%$ confidence level, according to the F-test, apart from the $\mathrm{Ni} \mathrm{He}-$ like $\mathrm{K} \alpha$ at $7.83 \mathrm{keV}$ which is significant at the 99.79\% confidence level. Besides the $\mathrm{He}-$ and $\mathrm{H}-$ like $\mathrm{Fe} \mathrm{K} \alpha$ lines $^{3}$, already discovered by ASCA (Ueno et al. 1994; Iwasawa et al. 1997; Bianchi et al. 2001), two lines at about 6.61 and $7.83 \mathrm{keV}$ are also detected with high significance. The energy of the first line points towards fluorescence from Be-like iron, which however has a quite low fluorescent yield of 0.11 (Krolik \& Kallman 1987). Moreover, the matter may likely be optically thick to the line, which results in an effective Auger destruction. It is therefore possible that this line is actually a blend of $\mathrm{Be}-$ and Li-like lines (the latter having an energy of about 6.65, a high fluorescent yield, 0.75 , and no possibility of Auger destruction). (The possibility that this line is the CS of the He-like iron line is ruled out by the low optical depth of the hot reflecting matter, as discussed below.) The line at $7.83 \mathrm{keV}$ is likely a blend of the $\mathrm{Ni} \mathrm{He}-$ like $\mathrm{K} \alpha(7.81 \mathrm{keV})$ and $\mathrm{Fe} \mathrm{He}-$ like $\mathrm{K} \beta$ lines $(7.87 \mathrm{keV})$. The rather wide range of ionization argues against collisional ionization. On the contrary, we have verified with CLOUDY 4 that photoionization may produce such a range in a single zone with e.g. a column density a few times $10^{21} \mathrm{~cm}^{-2}$ (see below) and a ionization parameter of about 150 .

The measured values of the $E W \mathrm{~s}$ can be compared with the results of the best previous observation, i.e. the $100 \mathrm{ks}$ ASCA observation (Bianchi et al. 2001). The results reported there are somewhat different, but were obtained with a simpler model. We refitted the ASCA spectrum with all the lines found by XMM-Newton (and the power law index fixed to 2.04) and found that, within the errors, the fluxes measured by ASCA are consistent with those measured by XMM-Newton. It should also be noted that the presence of the $6.61 \mathrm{keV}$ line vindicates the claim of Iwasawa et al. (1997), based on the first ASCA observation, of a redshift of the ionized lines.

The $E W$ of the $\mathrm{He}-$ and $\mathrm{H}$-like iron lines can be compared with the results of Matt et al. (1996) to derive at least an orderof-magnitude value for the column density of the reflecting

\footnotetext{
${ }^{3}$ Also for these lines the measured energies are not in agreement with the expected values, if only statistical errors are taken into account. However, in these cases identification is certain, as no other strong lines are present at those energies. It is worth noting that the energy of the He-like line is greater than expected, and that of the H-like line lower, ruling out inflow or outflow if the two lines, as likely, are produced in the same region.

${ }^{4}$ http://www . nublado.org/
}

material. In their Figs. 5 and 6 the $E W$ of these lines, calculated including contributions from both recombination and resonant scattering, are shown as a function of the column density of the reflecting material. The calculations assumed iron abundance and fractions equal to 1 of the considered ions. The measured iron overabundance roughly compensates for the less than one fraction of each ion (for instance, our CLOUDY calculation gives a value of 0.4 for the fraction of He-like iron atoms, and somewhat smaller value for the $\mathrm{H}$-like atoms). Even taking into account the fact that the ionized continuum includes also reflection from the warm reflector (Bianchi et al. 2001 estimated that the hot reflector contributes to about $3 / 4$ of the total ionized reflection), from the abovementioned figures of Matt et al. (1996) a column density of the hot ionized reflector of a few $\times 10^{21} \mathrm{~cm}^{-2}$ can be deduced.

\section{Summary}

We have analysed the high energy ( $>4 \mathrm{keV}$ ) XMM-Newton EPIC-pn spectrum of NGC 1068. The main results can be summarized as follows:

i) Possible (but not conclusive) evidence for variations of both neutral and ionized reflectors with respect to the December 1996 BeppoSAX observation is found, suggesting an upper limit to the size of both reflectors of a few parsecs. Another XMM-Newton observation, to compare results obtained with similar instruments, is however required to confirm this finding. It is worth noting that a variability on a time scale of about a year of the ionized reflector was found by Guainazzi et al. (2000) comparing two BeppoSAX observations, and variations on even shorter time scales were reported by Colbert et al. (2002).

ii) Iron is overabundant, with respect to lower $Z$ elements and when compared to the solar value, by a factor about 2 . Nickel is, in its turn, overabundant by a factor $\sim 2$ with respect to iron. A qualitatively similar result was found in the Circinus Galaxy (Molendi et al. 2003), where however both overabundances were smaller.

iii) The iron $\mathrm{K} \beta / \mathrm{K} \alpha$ flux ratio points to a low ionization state of iron, inconsistent with the $\mathrm{K} \alpha$ line energy, suggesting calibration problems. The nickel $\mathrm{K} \beta / \mathrm{K} \alpha$ is larger than expected, but the $\mathrm{K} \beta$ line may actually be a blend with ionized iron lines.

iv) The $\mathrm{Fe} \mathrm{K} \alpha$ Compton Shoulder is also detected, with a relative flux of 0.2 , in agreement with the value expected for reflection from Compton-thick matter (Matt 2002)

v) $\mathrm{Be}-, \mathrm{Li}-, \mathrm{He}-$ and $\mathrm{H}-$ like iron emission lines, as well as $\mathrm{He}-\mathrm{like} \mathrm{Ni}$ line, are also found. Their $E W$ suggests a column density of the hot ionized reflector of a few $\times 10^{21} \mathrm{~cm}^{-2}$ (Matt et al. 1996).

Acknowledgements. Based on observations obtained with XMM-Newton, an ESA science mission with instruments and contributions directly funded by ESA Member States and the USA (NASA). GM and SB acknowledge financial support from ASI.

\section{References}

Anders, E., \& Grevesse, N. 1989, Geo. Cosm. Acta, 53, 197

Antonucci, R. R. J., \& Miller, J. S. 1985, ApJ, 297, 621 
Band, D. L., Klein, R. I., Castor, J. I., \& Nash, J. K. 1990, ApJ, 362, 90

Bearden, J. A. 1967, Rev. Mod. Phys., 39/1, 78

Bianchi, S., Matt, G., \& Iwasawa, K. 2001, MNRAS, 322, 669

Colbert, E. J. M., Weaver, K. A., Krolik, J. H., Mulchaey, J. S., \& Mushotzky, R. F. 2002, ApJ, 581, 182

Elvis, M., \& Lawrence, A. 1988, ApJ, 331, 161

Ghisellini, G., Haardt, F., \& Matt, G. 1994, MNRAS, 267, 743

Guainazzi, M., Molendi, S., Vignati, P., Matt, G., \& Iwasawa, K. 2000, New Astron., 5, 235

Guainazzi, M., Matt, G., Antonelli, L. A., et al. 1999, MNRAS, 310, 10

Iwasawa, K., Fabian, A. C., \& Matt, G. 1997, MNRAS, 289, 443

House, L. L. 1969, ApJS, 18, 21

Kaastra, J. S., \& Mewe, R. 1993, A\&AS, 97, 443

Kinkhabwala, A., Sako, M., Behar, E., et al. 2002, ApJ, 575, 732
Koyama, K., Inoue, H., Tanaka, Y., et al. 1989, PASJ, 41, 731

Krolik, J. H., \& Kallman, T. R. 1987, ApJ, 320, L5

Matt, G., Brandt, W. N., \& Fabian, A. C. 1996, MNRAS, 280, 823

Matt, G., Guainazzi, M., Frontera, F., et al. 1997a, A\&A, 325, L13

Matt, G., Fabian, A. C., \& Reynolds, C. S. 1997b, MNRAS, 289, 175

Matt, G. 2002, MNRAS, 337, 147

Matt, G., Guainazzi, M., \& Maiolino, R. 2003, MNRAS, 342, 422

Molendi, S., Bianchi, S., \& Matt, G. 2003, MNRAS, 343, L1

Monier, R., \& Halpern, J. P. 1987, ApJ, 315, L17

Ogle, P. M., Brookings, T., Canizares, C. R., Lee, J. C., \& Marshall, H. L. 2003, A\&A, 402, 849

Sambruna, R., Netzer, H., Kaspi, S., et al. 2001, ApJ, 546, L13

Smith, D. A., \& Wilson, A. S. 2003, ApJ, 591, 138

Ueno, S., Mushotzky, R. F., Koyama, K., et al. 1994, PASJ, 46, L71

Young, A. Y., Wilson, A. S., \& Shopbell, P. L. 2001, ApJ, 556, 6 